\title{
Ophthalmoscopic findings in subacute sclerosing panencephalitis
}

\author{
S. H. GREEN* AND J. D. WIRTSGHAFTER
}

Departments of Neurology and Surgery (Ophthalmology), University of Kentucky Medical Center, Lexington, Kentucky, U.S.A.

Subacute sclerosing panencephalitis (SSPE) is a progressive central nervous system disorder affecting both grey and white matter; it affects children and young adults and, with only occasional exceptions, is fatal. Present interest in the illness stems from the observation that the disease is due to a slow virus infection produced by measles or a measles-like virus (Horta-Barbo:a, Fuccillo, and Sever, 1971). While several antiviral drugs have oे been tested, no specific therapy has so far been found (Haslam, McQuillen, and Clark, $\subseteq$ I 969$)$.

The incidence of SSPE is about one per million in the United States of America. The $\bigcirc$ male to female ratio is about $4: 1$ and the disease is more common in rural than urban areas. The patient may present with visual problems, behaviour disorders, and memory impairment; then there follows myoclonus and further deterioration including spast quadriparesis and dementia (Freeman, i 969). The electroencephalogram shows periodic complexes, the gamma globulins are raised in the cerebrospinal fluid, and thes measles antibody titres are chronically raised in both blood and cerebrospinal fluid. There is a relatively high incidence of SSPE in the Kentucky-Tennessee area of the United States (Jabbour, Garcia, Lemmi, Ragland, Duenas, and Sever, I969). Because many of these cases present initially with one or more ophthalmological problems, we have reviewed all twenty cases with this condition seen at the Neurology Department of the University of Kentucky Medical Center with particular reference to the ophthalmoscopic findings.

In some cases the ophthalmological examination may disclose findings which readily $\frac{\partial}{0}$ suggest a more diffuse neurological disease (Table I), but in other cases the findings

Table I Ophthalmological and ophthalmoscopic findings in subacute sclerosing panencephalitis which usually suggest the need for a neurological investigation

1. Gradual loss of visual acuity or visual field without ophthalmoscopic findings
2. Cortical blindness
3. Attacks of transient blindness
4. Deterioration of reading skills and school performance
5. Oedema of the optic disc (papilloedema or papillitis)
6. Optic atrophy or temporal pallor
7. Nystagmus or other abnormal ocular movements
8. Supranuclear gaze palsies
9. Ptosis 
suggest instead a more localized intraocular disease process (Table II).

Table II Ophthalmological and ophthalmoscopic findings in subacute sclerosing panencephalitis which suggest an ocular problem

I. Macular pigment epithelial abnormalities

2. Macular oedema

3. Macular haemorrhage

4. Retinochoroiditis

5. Gliotic retinal scar

6. Internal limiting membrane contracture

7. Serous detachment of the macula

8. Drusen of Bruch's mebrane

9. Pre-retinal opacities

\section{Material and methods}

Twenty cases of SSPE have been seen in the period 1966 to 197 I, mostly in the last 2 years, as the index of suspicion and our interest and knowledge of the disease have increased. The diagnosis has been confirmed in all the cases with the typical electroencephalographic pattern, raised cerebrospinal fluid gamma globulins, and raised measles antibody titres in blood and cerebrospinal fluid. Fourteen cases have been seen and personally examined by one or other of the authors, using direct and indirect ophthalmoscopy, the findings being documented by fundus photography with a hand-hel Kowa camera. The other six cases were reviewed from the hospital records and the Ophthalmolog Division photographic files. The age at onset varied from 3 to 18 years (median 9.5 ), and the mafo to female ratio was $3 \cdot 5: \mathrm{I}$.

Table III (overleaf) presents the clinical and ophthalmoscopic data of our patients. Cases I to 14 were specifically re-examined for the purposes of this study and are referred to as study patients. Table IV summarizes the incidence of specific ophthalmoscopic findings in the fourteen study patients and in the entire group of twenty patients.

Table IV Ophthalmoscopic findings in patients with subacute sclerosing panencephalitis

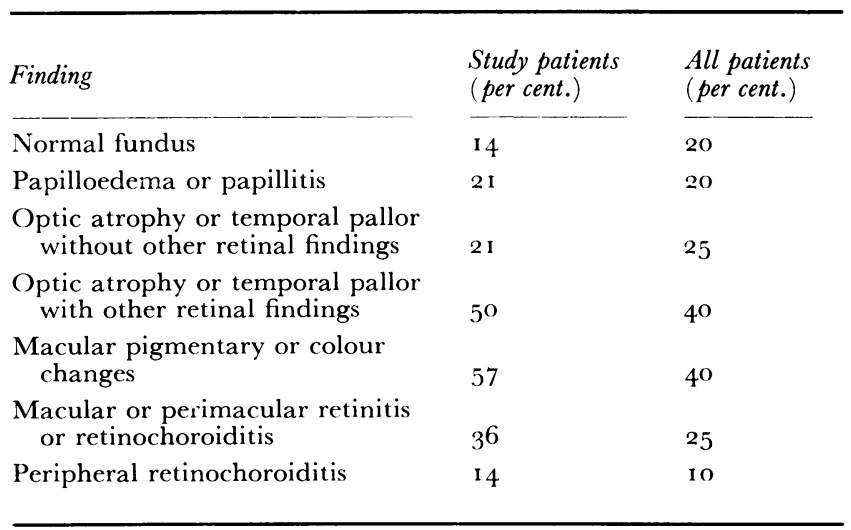

The columns do not add up to roo per cent. because some patients had more than one finding 
Table III Clinical data and ophthalmoscopic findings in twenty patients with subacute sclerosing panencep/

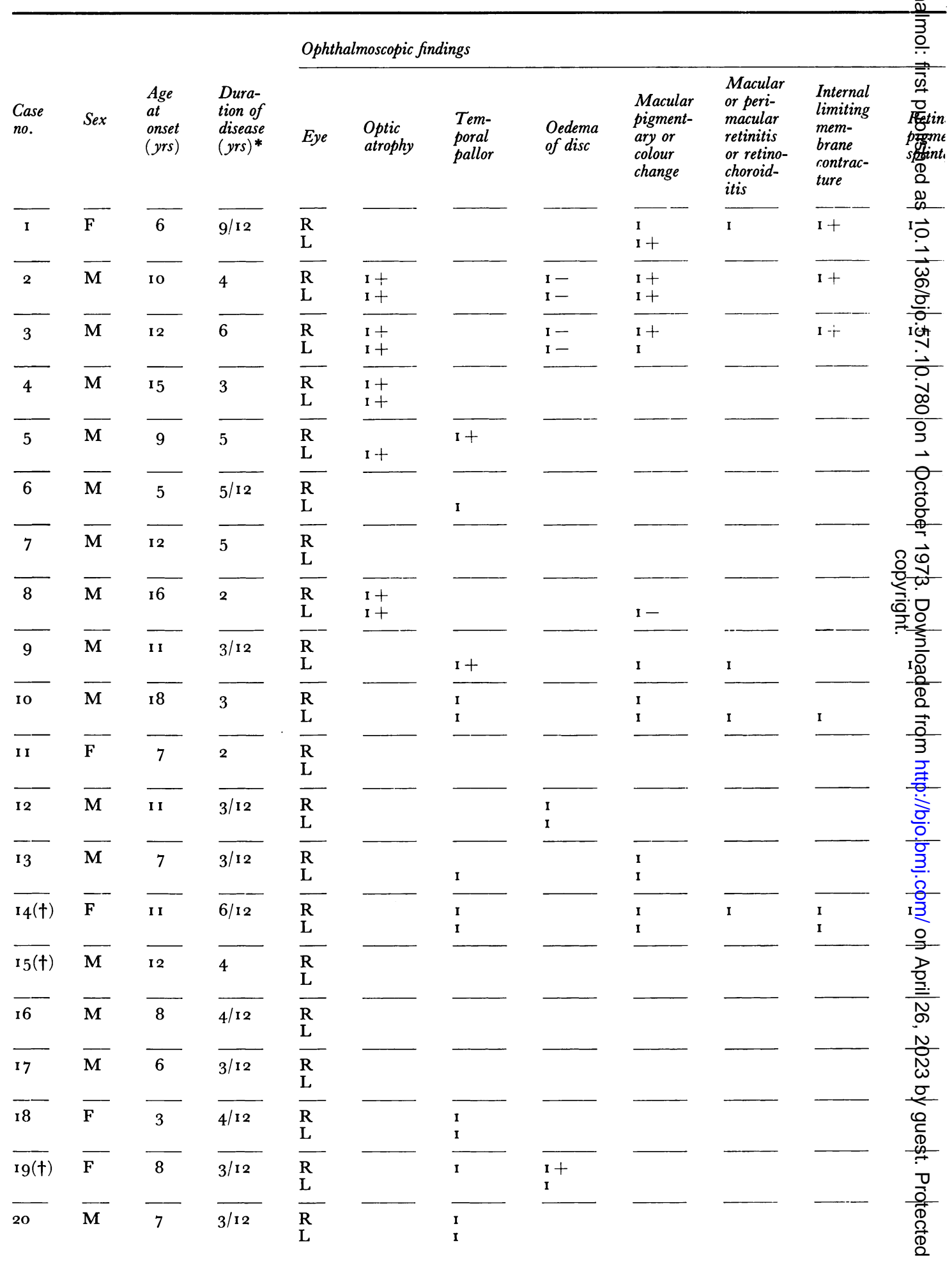


eri-

heral

tino-

loroid-

is

Small nerve fibre layer haemorrhage, choroidal vessel pattern not revealed

Other findings
Retinal vessels pulled into scar, slight preretinal scar covers pigment

Disc gliosis, red-orange macular colour, linear macular depigmentation

Disc gliosis

Disc gliosis

Disc gliosis, retinitis with pigmentation

Equatorial cluster of 3 white spots with black borders (?) compatible with histoplasmosis

Preretinal opacity, periarterial sheathing, intraretinal lipid deposits

Macular oedema noted for six weeks, vessel drawn into scar

Red-orange macular colour

examination $\quad-=$ Decreased from time of first examination 


\section{Results}

Our ophthalmoscopic findings are in general similar to those in the American and European literature (Hiatt, Grizzard, McNeer, and Jabbour, I97I; Robb and Watters, I970; Nelson, Weiner, Yanoff, and Deperalta, I970; Landers and Klintworth, I97 I Karel, Otradovec, Peleška, and Nevšmal, ı 97ı; Otradovec, ı969; Collard, Payeur, Zenglien, Eber, Schupp, and Bronner, I 97 I ; Perdriel, Cornard, Bouvier, and Delpuget, I 969; Glowacki, Guazzi, and van Bogaert, I967). Papilloedema, papillitis, optic atrophy, and temporal pallor may all be present; in addition to disturbances of ocular motility, visual perception disorders, and cortical blindness, these are apt to suggest to the ophthalmologist the need for further neurological evaluation. For a number of reasons it proved difficult to separate papilloedema from papillitis and these two conditions with oedema of the optic disc were considered together.

However, in our patients (Table IV) and those reported in the literature, the ophthalmoscopic findings often suggested a localized ocular problem such as an inherited retinal dystrophy or a retinitis which might have delayed recognition of the more widespread nature of the disease. This is particularly true when the disorder is confined to the macular or perimacular region and the ophthalmic features are present months before the neurological signs are obvious. These abnormalities in the macula in their minimal form consisted of granular pigmentary changes or red-orange colour changes which were often bilaterally symmetrical. However, gliotic macular or perimacular scarring was always unilateral and associated with the pigmentary changes in the ipsilateral or the contralateral eye (Fig. I). The loss of pigment epithelium was never so severe as to reveal the underlying choroidal vessel pattern.

A typical example of a macular scar is shown in Fig. 2. The size is about one to two disc diameters, there is no significant vitreous opacity, the surrounding retina shows radiating folds indicating contracture of the internal limiting membrane, and there is hyperpigmentation in the form of pigment splinters radiating from the edges of the gliotic

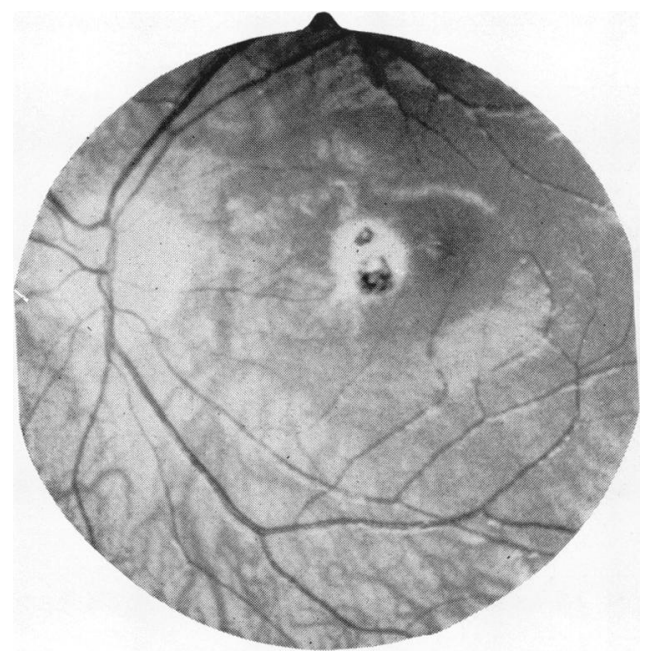

FIG. I Fundus photograph of left eye, Ciase 9, showing perimacular depigmented and hyperpigmented scar. $\mathcal{N o}$ underlying choroidal vessels are seen

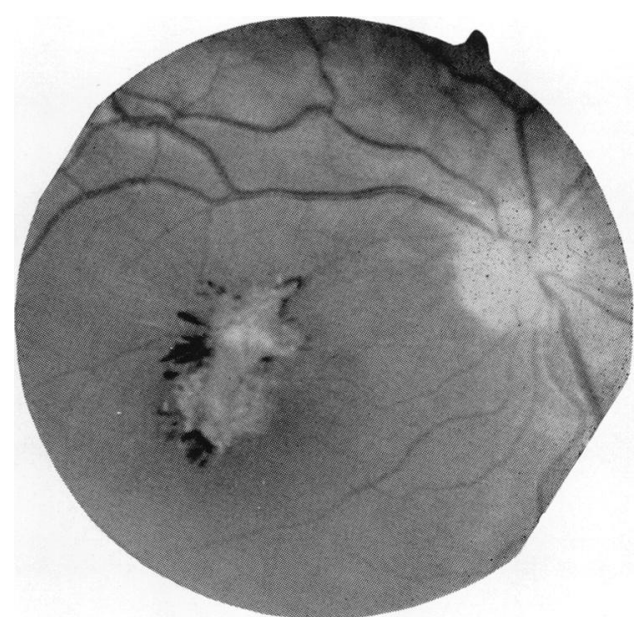

FIG. 2 Fundus photograph of right eye, Ciase 3, showing pigment-splinters and contracture of internal limiting membrane of retina 
scar. There are no satellite lesions. The retinal vessels within the scar are obscured.

Fig. 3 demonstrates the dragging of adjacent retinal vessels towards the scar in a perimacular lesion.

Fig. 4 shows features not seen in the other cases. There is a mild pre-retinal haze, with a lipid-like infiltration of the macular region and periarteriolitis. Such vascular sheathing has not previously been reported in the literature, and Otradovec (I969) particularly commented on the absence of vascular sheathing and SSPE.

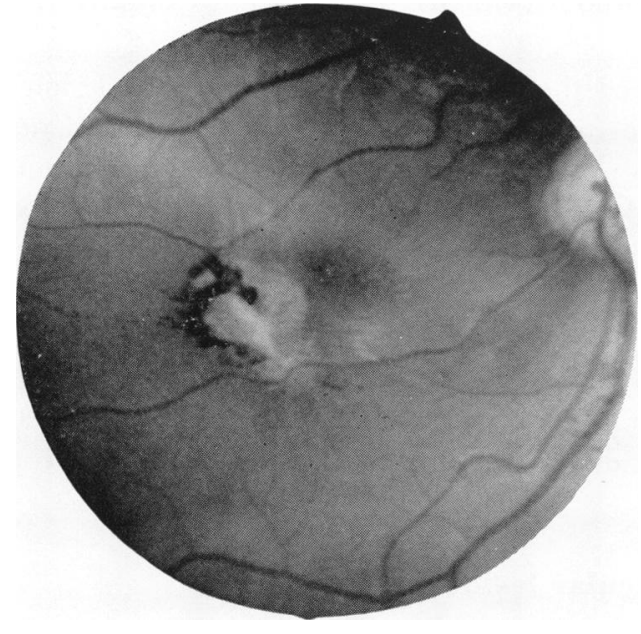

FIG. 3 Fundus photograph of right eye, Case 14, showing retinal vessels pulled into the gliotic perimacular scar. Peripheral hyperpigmentation with some pigmentsplinters are also seen

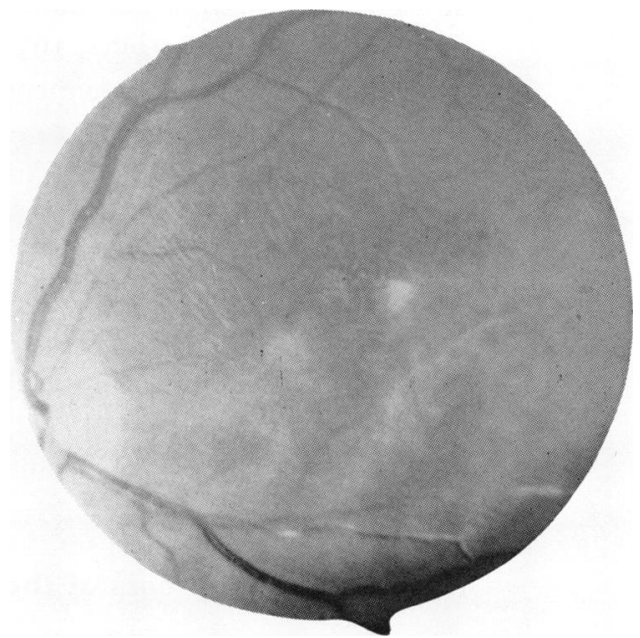

FIG. 4. Fundus photograph of left eye, Case Io, showing pre-retinal opacity, periarterial sheathing, and intraretinal lipidlike deposits

In two other patients a peripheral retinochoroiditis was also present.

\section{Discussion}

The ophthalmoscopic findings in one-fifth of our study patients showed oedema of the optic disc and another one-fifth showed only optic atrophy or temporal pallor which would have suggested a need for a neurological evaluation (Table IV). But over half the patients had ophthalmoscopic findings which would suggest a primary ocular disease. In order to help the ophthalmologist recognize SSPE, the characterization of the macular lesion and its differential diagnosis will be discussed.

The macular and perimacular abnormalities begin within the retinal pigment epithelium (granularity, red-orange colour change) and neuroepithelium (oedema) haemorrhage). Involvement of the choroid is limited to the choriocapillaris and may mimic the appearance of a central serous retinopathy (Otradovec, I969). As the disease progresses, the retinitis may subside or become more severe, but spread to the vitreous cavity is limited to minimal pre-retinal haze or snowflake-like opacities. Ultimately a gliotic retinal scar may develop with contracture of the internal limiting membrane. A macular hole may develop in the centre of the lesion. These retinal scars do not have satellite lesions and are not bilaterally symmetrical. While hyperplastic pigmentary changes may be present centrally within the scar, they are more often present at the edges of the lesion and have a splinterlike appearance. 
Retinal vessel involvement is usually limited to obscuration of the vessels within the scar. Only one of our eyes showed retinal periarterial sheathing. In two eyes the course of the nearby retinal vessels was deviated into the scar. Anastomosis of the retinal and choroidal vessels has not been seen. Fluorescein studies have shown capillary occlusions in the retinal vessels surrounding the macula.

The limited involvement of the choroid is indicated by the observation that the choroidal vessels were not exposed by the lesion. Fluorescein studies further suggest focal defects in the retinal pigment epithelium without evidence of significant exudative lesions of the choroid (Karel and others, I97I).

Pathological studies have confirmed the clinical impression that the lesion of SSPE is primarily a retinitis. Electron microscopic examination of the eye of a patient with SSPE has shown microtubular structures compatible with viral nucleocapsids present within the retinal neuroepithelium (Landers and Klintworth, I97 I). Light microscopic examinations have shown that the process is primarily a retinitis of the neural and pigment epithelium and that choroidal involvement is limited to the choriocapillaris (Nelson and others, I970).

It is important that these pathological observations be extended to the clinical description of the disease so that SSPE is considered with the differential diagnosis of retinal disease and not inappropriately dismissed as another rare cause of chorio-retinitis. The macular lesions of SSPE should be described as a retinitis, or, when appropriate, as a retinochoroiditis.

\section{Differential diagnosis of the macular lesions}

Table $\mathrm{V}$ shows some of the diseases of the macular region which may resemble the changes seen in SSPE. The classification of heredo-degenerations follows that of Deutman (I97 I).

Over half our patients had macular pigmentary or colour changes. These are most likely to be misinterpreted as due to one of the heredo-degenerations of the retinal neuroepithelium. The granular pigmentary changes seen in SSPE because of the extension of the disease to the retinal pigment epithelium could be likewise misinterpreted as being due to hereditary disorders of the retinal pigment epithelium, in particular vitelliform dystrophy of the fovea-Best's disease (Rousseau and Tasman, I97I). Inflammatory diseases, in particular toxoplasmosis, can provide diagnostic problems. In toxoplasma exudative retino-choroiditis, there is often a marked vitreous cellular response which may be associated with a focal panophthalmitis (VanMetre, Knox, and Maumenee, I964; Maumenee, i 966).

\section{Summary and conclusions}

(I) Twenty children with SSPE were reviewed with respect to ophthalmoscopic findings, and 75 per cent. of them were found to have some abnormality.

(2) The changes thought to be most typical but not necessarily pathognomonic were granularity, oedema, scarring of the macula with pigment splinters at the edge of the lesion, contraction of the internal limiting membrane, and dragging of the retinal blood vessels towards the scar.

(3) The lesion is best described as a retinitis or, when the choriocapillaris is involved, as a retino-choroiditis.

(4) Because ophthalmological features may present before marked neurological signs are present, SSPE must be considered in the differential diagnosis of children with retinitis, oedema of the optic disc, optic atrophy, or more complex disorders of visuomotor function. 
Table $\mathbf{V}$ Some diseases of the macular region which may resemble the changes seen in subacute sclerosing panencephalitis. The diseases are classified on the basis of the ocular structures primarily and mainly affected

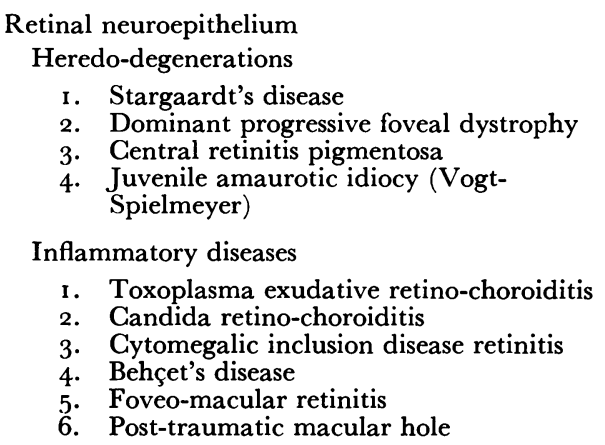

Retinal pigment epithelium

Heredo-degeneration

I. Vitelliform dystrophy of the fovea

Inflammatory diseases

I. Rubella retinopathy

2. Acute posterior multifocal placoid pigment epitheliopathy
Bruch's membrane

Heredo-degenerations

I. Dominant drusen (hyaline dystrophy)

2. Pseudo-inflammatory foveal dystrophy (Sorsby)

\section{Choriocapillaris}

Idiopathic

I. Central serous choroidopathy

We are grateful to D. B. Clark, M.D., Ph.D., Professor and Chairman of the Department of Neurology, under whose care the patients were admitted. The histories of these patients were collected as a part of a larger study of SSPE by P. G. Procopis, M.B., M.R.A.C.P. Mrs. Mary Leonidakis, Social Worker, helped to arrange for the examination of these patients, many of whom lived in inaccessible areas of Appalachia. Wm. Richard Green, M.D. kindly discussed the interpretation of some of the fundus photographs.

\section{References}

COllard, M., PAYeUr, G., Zenglein, J. P., EBER, A. M., SchupP, G., and Bronner, A. (I97i) Rev. Otoneuro-ophtal., 43, 25

Deutman, A.F. (I97 I) “The Hereditary Dystrophies of the Posterior Pole of the Eye". Van Gorcum, Assen freeman, J. M. (1969) $\mathcal{J}$. Pediat., 75, 590

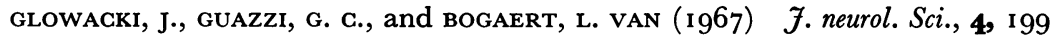

hASLAM, R. H. A., McQuillen, M. P., and ClARK, D. B. (1969) Neurology (Minneap.), r9, Io8o

hiatt, R. L., Grizzard, H. T., maneer, P., and Jabbour, J. T. (i97I) Trans. Amer. Acad. Ophthal.

Otolaryng., 75, 344

horta-barbosa, l., fuccillo, D. A., and sever, J. l. (i97i) J. Amer. med. Ass., 218, i I85

Jabbour, J. T., GARcia, J. H., Lemmi, H., RAgland, J., DUenas, D. A., and Sever, J. L. (I96o) Ibid., 207,2248

KARel, I., Otradovec, J., PelešKa, M., and nevšimal, O. (197I) Ophthalmologica (Basel), 162, 348

LANDERS, M. B., and Klintworth, G. K. (I97 I) Arch. Ophthal. (Chicago), 86, I 56

maumenee, A. E. (I g66) In "Retinal Diseases": "Symposium on Differential Diagnostic Problems

of Posterior Uveitis", ed. S. J. Kimura and W. M. Caygill, p. 28r. Lea and Febiger, Philadelphia nelson, D. A., Weiner, A., Yanoff, m., and deperalta, J. (i970) Arch. Ophthal. (Chicago), 84, 6ri3 OTRADOVEC, J. (1969) Ophthalmologica (Basel), r57, 427

PERdriel, G., CORnANd, G., Bouvier, s., and Delpuget, J. (I969) Bull. Soc. Ophtal. Fr., p. I067 robB, R. M., and watters, G. v. (1970) Arch. Ophthal. (Chicago), 83, 426

rousseau, A., and tasman, w. (197I) In "Retinal Diseases in Children", ed. W. Tasman, p. I9.

Harper and Row, New York

van metre, t. E., knox, D. L., and maumenee, A. E. (I964) Amer. 7. Ophthal., 58, 6 